\begin{tabular}{|c|c|c|c|c|c|c|}
\hline \multirow{4}{*}{ Impact Factor: } & ISRA (India) & $=3.117$ & SIS (USA) & $=0.912$ & ICV (Poland) & $=6.630$ \\
\hline & ISI (Dubai, UAE & $=0.829$ & РИНЦ (Russia & $=0.156$ & PIF (India) & $=1.940$ \\
\hline & GIF (Australia) & $=0.564$ & ESJI (KZ) & $=8.716$ & IBI (India) & $=4.260$ \\
\hline & JIF & $=1.500$ & SJIF (Morocco & $=\mathbf{5 . 6 6 7}$ & OAJI (USA) & $=0.350$ \\
\hline
\end{tabular}

\section{SOI: 1.1/TAS DOI: 10.15863/TAS International Scientific Journal Theoretical \& Applied Science}

\author{
p-ISSN: 2308-4944 (print) e-ISSN: 2409-0085 (online) \\ Year: 2019 Issue: $04 \quad$ Volume: 72
}

Published: 08.04.2019 http://T-Science.org
QR - Issue

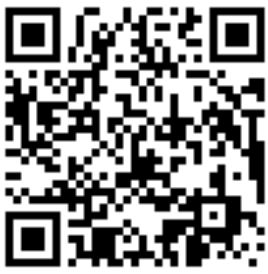

Kanagat Skakovna Abdiramasheva lecturer of Propedeutics and Internal disease department

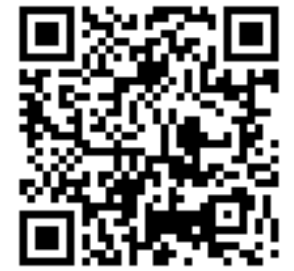

Yassavi, Kazakhstan

abdiramasheva65@mail.ru

\title{
GLUCOCORTICOSTEROIDS AND DEVELOPMENT OF DIABETES MELLITUS (literature review)
}

Abstract: This article presents a review of the literature on the study of the development of diabetes mellitus when using glucocorticosteroids in the treatment of various diseases. Along with this, there have been studied some mechanisms of the development of an increase in the level of glucose in patients taking steroid drugs for the treatment of certain diseases.

Key words: glucocorticosteroids, hyperglycemia, diabetes mellitus, corticosteroids.

Language: Russian

Citation: Abdiramasheva, K. S. (2019). Glucocorticosteroids and development of diabetes mellitus. literature review. ISJ Theoretical \& Applied Science, 04 (72), 15-19.

Soi: http://s-o-i.org/1.1/TAS-04-72-3 Doi: crossef https://dx.doi.org/10.15863/TAS.2019.04.72.3

\section{ГЛЮКОКОРТИКОСТЕРОИДЫ И РАЗВИТИЕ САХАРНОГО ДИАБЕТА \\ (литературный обзор)}

Аннотация: В данной статье представлен обзор литературы по изучению развития сахарного диабета при использовании глюкокортикостероидов в лечении различных заболеваний. Наряду с этим, изучены некоторые механизмы развития повышения уровня глюкозы у пациентов принимающих стероидные препараты для лечения тех или иных заболеваний.

Ключевые слова: глюкокортикостероиды, гипергликемия, сахарный диабет, кортикостероиды.

\section{Введение.}

Стероиды - это лекарства, которые широко используются для лечения многих заболеваний. Хотя, глюкокортикоиды часто назначаются как противовоспалительные и иммунодепрессивные препараты, они имеют несколько побочных эффектов, и гипергликемия представляет собой одну из наиболее распространенных. Стероиды являются основной причиной гипергликемии, вызванной лекарственными препаратами. Они не только усугубляют гипергликемию у пациентов с сахарным диабетом (СД), но также вызывают СД у здоровых пациентов, причем частота может достигать до 46\%. Кроме того, в некоторых случаях они могут вызывать острые осложнения, такие как гиперосмолярное состояние и диабетический кетоацидоз, и даже летальный исход, особенно у пациентов с уже существующим СД [1]. Эффекты глюкокортикоидов на метаболизм глюкозы включают подавление транспортера глюкозы в мышцах, который увеличивает количество инсулина, необходимого для поглощения глюкозы клетками, увеличение выработки глюкозы в печени, ингибирование связывания инсулина рецептором и снижение секреции инсулина в островковых клетках. Следовательно, введение глюкокортикоидов может усугубить предиабет или недиагностированный диабет и может трансформировать умеренный диабет в клинически тяжелое заболевание, что может привести к гипергликемической гиперосмолярной коме. Однако некоторые симптомы гипергликемии, такие как жажда, сухость во рту, слабость, потеря веса и полиурия также часто встречаются у пациентов [2]. Таким образом, гипергликемия выявленная у пациента требует дифференциальной диагностики, так как причиной повышение уровня глюкозы могут быть 


\begin{tabular}{|c|c|c|c|c|c|c|}
\hline \multirow{4}{*}{ Impact Factor: } & ISRA (India) & $=3.117$ & SIS (USA) & $=0.912$ & ICV (Poland) & $=6.630$ \\
\hline & ISI (Dubai, UAE & $=0.829$ & РИНЦ (Russia & $=\mathbf{0 . 1 5 6}$ & PIF (India) & $=1.940$ \\
\hline & GIF (Australia) & $=0.564$ & ESJI (KZ) & $=8.716$ & IBI (India) & $=4.260$ \\
\hline & JIF & $=1.500$ & SJIF (Morocco & $=5.667$ & OAJI (USA) & $=0.350$ \\
\hline
\end{tabular}

лекарственные препараты применяемые для лечения определенных заболеваний.

\section{Цель исследования.}

Целью исследования явилась изучение данных литературы по развитию сахарного диабета после приема глюкокортикостероидов, а также оценить основной механизм ответственный за повышение уровня глюкозы при лечении стероидными препаратами.

\section{Методы исследования.}

В данной статье проведен обзор литературы по ключевым словам «стероид-индуцированная гипергликемия», «глюкокортикостероиды и гипергликемия», «глюкокортикостероиды и диабет» в базах данных PubMed и ScienceDirect. При вводе ключевых слов наблюдается увеличение публикации с 1973 по 2018 гг, что показано в рисунке 1.

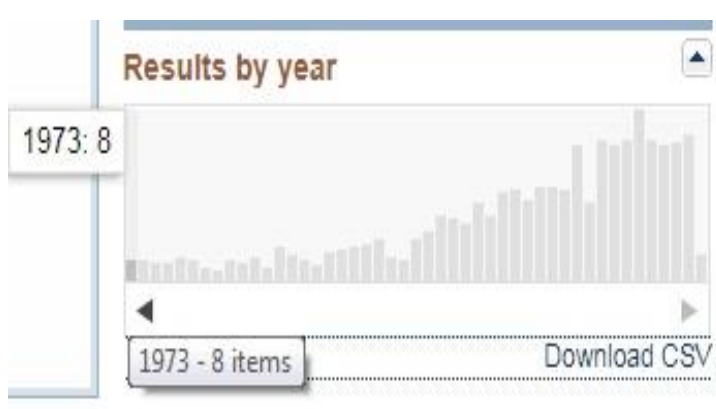

a)

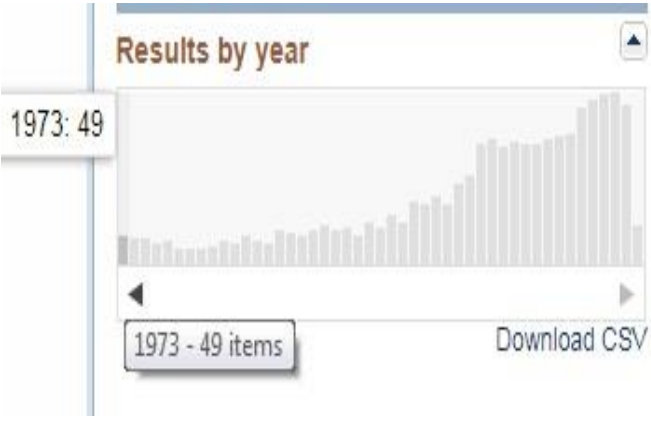

б)

Рисунок 1. Количество научных статьей в базе РubМеd при вводе ключевых слов: а)
«люкокортикостероиды и гипергликемия», б) «глюкокортикостероиды и диабет».

Результаты литературного обзора.

Глюкокортикоидные гормоны секретируются корой надпочечников под контролем гипоталамо-гипофизарнонадпочечниковой системы. Глюкокортикостероиды - это гормоны стресса, которые облегчают реакцию защиты организма путем увеличения производство глюкозы в печени, липолиза жировой ткани и протеолиза, а также поддержание адекватного артериального давления. В клинике синтетические кортикостероиды широко используются в лечении многочисленных заболеваний в связи с их мощным противовоспалительным и иммуносупрессивным действием. В 1908 году впервые было установлено, что «вещества» секретируемые надпочечниками вовлечены в метаболизм глюкозы, а последующие исследования на животных с адреналэктомией, выявили развитие гипогликемии [3].

С момента появления глюкокортикоидной терапии аутоиммунных заболеваний в 1940-х годах их широкое применение привело к одновременному ограничению использования и обнаружению многих побочных метаболических эффектов [4]. Пациенты получают стероидную терапию при лечении множества состояний, от легочных до ревматологических и дерматологических заболеваний. Известно, что стероиды вызывают гипергликемию, которая возникает в период, пока пациент принимает стероиды. Если пациент не страдающий СД находится на пятидневном курсе стероидами, мониторинг уровня глюкозы в крови обычно не выполняется. Однако, если пациенту требуются стероиды в течение длительного периода времени, обычно порядка нескольких недель или месяцев, целесообразно контролировать уровень глюкозы в крови [5]. Так, Нoes J.N. и соавторы выявили, что у пациентов длительно принимающих кортикостероиды наблюдается пониженная чувствительность к инсулину и функция $\beta$-клеток по сравнению с контрольной группой [6].

По данным литературы распространенность СД вследствие перорального приема глюкокортикоидов колеблется от $2 \%$ до $50 \%$. Имеются несколько факторов риска развития стероид-индуцированного СД (СИСД). Большая длительность, высокая доза и тип непрерывной терапии глюкокортикоидами, пожилой возраст, индекс массы тела, гестационный СД, семейный анамнез диабета и уровень гликолизированного гемоглобина являются факторами риска развития СИСД. При этом, предиабет также является предполагаемым фактором риска. Важно идентифицировать пациентов, подвергающихся 


\begin{tabular}{|c|c|c|c|c|c|c|}
\hline \multirow{4}{*}{ Impact Factor: } & ISRA (India) & $=3.117$ & SIS (USA) & $=0.912$ & ICV (Poland) & $=6.630$ \\
\hline & ISI (Dubai, UAE & $=0.829$ & РИНЦ (Russia) & $=0.156$ & PIF (India) & $=1.940$ \\
\hline & GIF (Australia) & $=0.564$ & ESJI (KZ) & $=8.716$ & IBI (India) & $=4.260$ \\
\hline & JIF & $=1.500$ & SJIF (Morocco & $=5.667$ & OAJI (USA) & $=0.350$ \\
\hline
\end{tabular}

лечению стероидами, которым грозит СИСД, так как диабет может остаться незамеченным у пациентов с нормальным уровнем глюкозы [7,8,9].

Пациенты из группы риска должны быть обследованы на наличие гипергликемии в течение первых 1-2 дней стероидной терапии, когда развивается большинство случаев СИСД. Стероиды вызывают в основном постпрандиальную гипергликемию, поэтому показатели глюкозы в крови после приема пищи более чувствительны, чем тесты на глюкозу натощак. СИСД лучше всего диагностируется пероральным тестом на толерантность к глюкозе [5].

Механизмы, лежащие в основе этих так называемых диабетогенных эффекты глюкокортикоидов в отношении метаболизма глюкозы, липидов и белков были изучены в 1960- 1970-х годах и были в основном связаны с глюкокортикоид-индуцированной

резистентностью к инсулину на уровне печени, скелетных мышц и жировой ткани [3]. Существует ряд механизмов, объясняющих повышение уровня глюкозы в крови вследствие терапии стероидами. Глюкокортикоиды усиливают глюконеогенез в печени и вызывают повышение резистентности к инсулину. Они также вызывают распад белков и липидов, тем самым обеспечивая субстратом процесс глюконеогенеза. После введения глюкокортикоидов наблюдается подавление периферического поглощения глюкозы скелетными мышцами. Это объясняет причину, по которой глюкокортикоиды вызывают начальную постпрандиальную гипергликемию $[3,10,11]$.

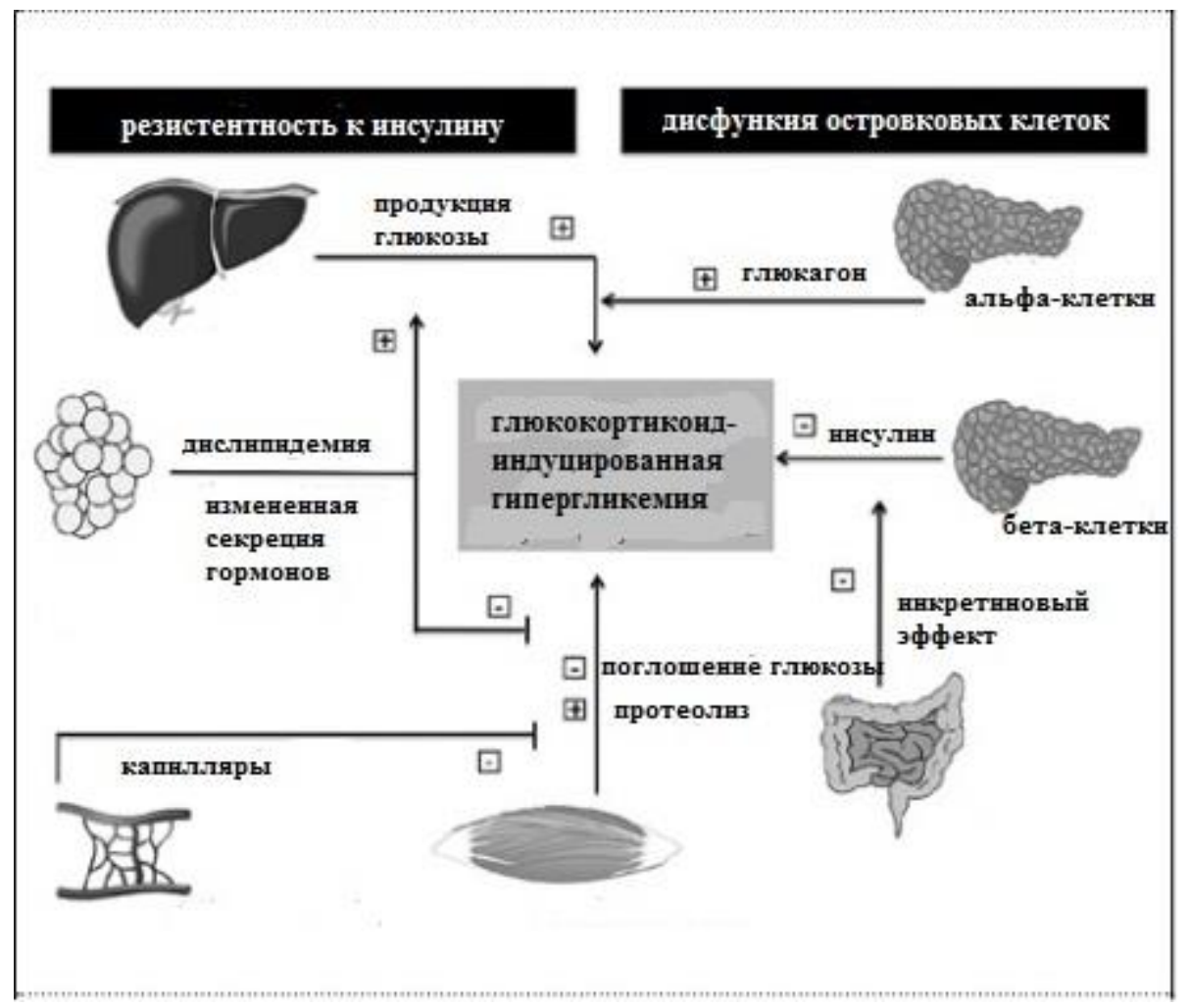

Рисунок 2. Механизм развития глюкокортикоид-индуцированной гипергликемии [3].

Dănescu A.S. и соавторы считают, что влияние стероидов на метаболизм глюкозы является результатом нескольких путей, в том числе, дисфункции бета-клеток, снижение аффинности связывания инсулина или уменьшение числа рецепторов, ингибирование каскадов постинсулиновых рецепторов [12,13].

Кроме того, по мнению Rafacho А. и соавторов, кортикостероиды могут стимулировать абдоминальное ожирение, повышать содержание в плазме жирных кислот и триглицеридов. В ответ на это, в попытке поддержать нормогликемию, $\beta$ клетки поджелудочной железы претерпевают несколько морфофункциональных адаптаций, которые приводят к гиперинсулинемии. Неспособность $\quad \beta$-клеток компенсировать эту ситуацию способствует нарушению гомеостаза глюкозы, что может приводит к гипергликемии. Лечение кортикостероидами не только изменяет функцию $\beta$-клеток поджелудочной железы, но также влияет 


\begin{tabular}{|c|c|c|c|c|c|c|}
\hline \multirow{4}{*}{ Impact Factor: } & ISRA (India) & $=3.117$ & SIS (USA) & $=0.912$ & ICV (Poland) & $=6.630$ \\
\hline & ISI (Dubai, UAE & $=0.829$ & РИНЦ (Russia & $=0.156$ & PIF (India) & $=1.940$ \\
\hline & GIF (Australia) & $=0.564$ & ESJI (KZ) & $=8.716$ & IBI (India) & $=4.260$ \\
\hline & JIF & $=1.500$ & SJIF (Morocco & $=\mathbf{5 . 6 6 7}$ & OAJI (USA) & $=0.350$ \\
\hline
\end{tabular}

на их действие, которое может привести к гиперглюкагонемии, что также способствует нарушению гомеостаза глюкозы и гипергликемии [14].

Проблемы в лечении стероидиндуцированного диабета связаны с широкими колебаниями постпрандиальной гипергликемии и отсутствием четко определенных протоколов лечения. Основой лечения является инсулинотерапия, совмещенная с диетотерапией. Инсулин является лечением выбора в большинстве случаев СИСД. Поскольку, при приеме кортикостероидов снижают дозу препаратов, эндокринная функция организма пациентов обычно возвращается к исходному уровню и потребность в инсулине снижается. Долгосрочное использование низких доз глюкокортикоидов было связано с повышенной сердечно-сосудистой смертностью, возможно, из-за микрососудистых осложнений гипергликемии. Поэтому своевременная идентификация СИСД имеет решающее значение, при котором, факторы риска сердечно-сосудистых заболеваний могут быть оптимизированы и достигнут адекватный гликемический контроль $[4,15]$.

Лечение СИСД фокусируется на двух основных направлениях. В начальный период глюкокортикоиды оказывают преобладающее влияние на уровень глюкозы в крови после приема пищи, исходя из этого первый механизм лечения направлен на борьбу с СИСД - это увеличение болюсного инсулина до приема пищи для того, чтобы предотвратить постпрандиальную гипергликемию. Использование этого базальноболюсного подхода лечения СИСД хорошо известно и есть доказательства того, что он является очень эффективным. Альтернативный подход заключается в том, что инсулин вводится одновременно с глюкокортикоидами, тогда можно уменьшить гипергликемический эффект глюкокортикоидов [8].

\section{Вывод.}

Таким образом, кортикостероиды могут быть причиной гипергликемии и сахарного диабета у пациентов принимающих данные препараты для лечения тех или иных заболеваний. Риск гипергликемии и СД возрастает у пациентов находящихся на длительном лечении стероидными препаратами, что требует мониторинга гликемического профиля для предупреждения возникновения осложнений диабета.

\section{References:}

1. Tamez-Pérez, H. E., Quintanilla-Flores, D. L., Rodríguez-Gutiérrez, R., GonzálezGonzález, J. G., \& Tamez-Peña, A. L. (2015). Steroid hyperglycemia: Prevalence, early detection and therapeutic recommendations: A narrative review. World J Diabetes, 6(8), 10731081.

2. Jeong, Y., et al. (2016). A Pilot Study Evaluating Steroid-Induced Diabetes after Antiemetic Dexamethasone Therapy in Chemotherapy-Treated Cancer Patients. Cancer Res Treat, 48(4), 1429-1437.

3. Van Raalte, D. H., \& Diamant, M. (2014). Steroid diabetes: from mechanism to treatment? Neth J Med, 72(2), 62-72.

4. Hwang, J. L., \& Weiss, R. E. (2014). Steroidinduced diabetes: a clinical and molecular approach to understanding and treatment. Diabetes Metab Res Rev, 30(2), 96-102.

5. Byrd, V., \& Nemeth, A. (2018). Steroidinduced Diabetes Complicating Treatment of Epidermolysis Bullosa Acquisita: A Preventable Treatment Complication Stresses the Importance of Primary Care Follow-up. Cureus, 10(11), e3608.

6. Hoes, J. N., et al. (2011). Glucose tolerance, insulin sensitivity and $\beta$-cell function in patients with rheumatoid arthritis treated with or without low-to-medium dose glucocorticoids. Ann Rheum Dis, 70(11), 1887-1894.

7. Gonzalez-Gonzalez, J. G., et al. (2013). Hyperglycemia related to high-dose glucocorticoid use in noncritically ill patients. Diabetol Metab Syndr, 5, 18.

8. Clore, J. N., \& Thurby-Hay, L. (2009). Glucocorticoid-induced hyperglycemia. Endocr Pract, 15(5), 469-474.

9. Kim, S. Y., et al. (2011). Incidence and risk factors of steroid-induced diabetes in patients with respiratory disease. J Korean Med Sci, 26(2), 264-267.

10. Lakhani, O. J., Kumar, S., Tripathi, S., Desai, M., \& Seth, C. (2017). Comparison of Two Protocols in the Management of Glucocorticoid induced Hyperglycemiaamong Hospitalized 


\begin{tabular}{|c|c|c|c|c|c|c|}
\hline \multirow{4}{*}{ Impact Factor: } & ISRA (India) & $=3.117$ & SIS (USA) & $=0.912$ & ICV (Poland) & $=6.630$ \\
\hline & ISI (Dubai, UAE & $=0.829$ & РИНЦ (Russia) & $=0.156$ & PIF (India) & $=1.940$ \\
\hline & GIF (Australia) & $=0.564$ & ESJI (KZ) & $=8.716$ & IBI (India) & $=4.260$ \\
\hline & JIF & $=1.500$ & SJIF (Morocco & $=5.667$ & OAJI (USA) & $=0.350$ \\
\hline
\end{tabular}

Patients. Indian J Endocrinol Metab, 21(6), 836844.

11. Iwamoto, T., Kagawa, Y., Naito, Y., Kuzuhara, S., \& Kojima, M. (2004). Steroidinduced diabetes mellitus and related risk factors in patients with neurologic diseases. Pharmacotherapy, 24(4), 508-514.

12. Dănescu, A. S., et al. (2018). Immunological markers as predictors of developing steroidinduced diabetes mellitus in pemphigus vulgaris patients: An observational study. Medicine (Baltimore), 97(17), e0463.

13. Ruzzin, J., Wagman, A. S., \& Jensen, J. (2005). Glucocorticoid-induced insulin resistance in skeletal muscles: defects in insulin signalling and the effects of a selective glycogen synthase kinase-3 inhibitor. Diabetologia, 48(10): 21192130.

14. Rafacho, A., Ortsäter, H., Nadal, A., \& Quesada, I. (2014). Glucocorticoid treatment and endocrine pancreas function: implications for glucose homeostasis, insulin resistance and diabetes. $J$ Endocrinol, 223(3), 49-62.

15. Hoogwerf, B., \& Danese, R. D. (1999). Drug selection and the management of corticosteroidrelated diabetes mellitus. Rheum Dis Clin North Am, 25(3), 489-505. 